\title{
Consensos y disensos en torno al concepto de naturaleza de la ciencia (nos) en la comunidad iberoamericana de didáctica de las ciencias*
}

\author{
Consensus and dissent around the concept of nature of scien- \\ $c e$ (nos) in the ibero-american community of science education \\ Acordos e desacordos em torno do conceito da natureza da ciên- \\ cia (nos) ibero-americana de ensino da comunidade da ciência
}

Recibido: diciembre de 2011 Aceptado: junio de 2012
Rafael Yecid Amador Rodríguez

Agustín Adúriz-Bravo

\begin{abstract}
Resumen
El objeto de este documento es presentar los resultados de una investigación referida a algunos acuerdos y desacuerdos en torno al concepto de "naturaleza de la ciencia" (conocido internacionalmente con el acrónimo inglés NOS) que aparecen entre especialistas de la didáctica de las ciencias naturales; específicamente, nos concentramos en aquellos pertenecientes a Iberoamérica. Para ello tuvimos en cuenta la producción académica publicada en artículos de revistas especializadas, en el periodo comprendido entre el año 2000 y el 2009; buscamos artículos que desarrollaran algún tipo de relación sustantiva con la línea de investigación NOS. El principal hallazgo de nuestro trabajo consiste en que, hoy en día, la NOS es un campo de reflexión e innovación didáctica claramente reconocible para los investigadores iberoamericanos de didáctica de las ciencias.
\end{abstract}

Palabras clave: naturaleza de la ciencia, epistemología, metaciencias, alfabetización científica, currículo de ciencias, Iberoamérica.

\begin{abstract}
The purpose of this paper is to present the results of an investigation referred to some agreements and disagreements on the concept of 'nature of science' (known internationally with the acronym NOS) that appear between specialists in the teaching of natural sciences; specifically, we focus on those from Latin America. To do this we considered the academic production in the form of journal articles, released between 2000 and 2009, we seek articles that develop some kind of substantive relationship with the research NOS. The main finding of this study is that, today, the NOS is a field of reflection and didactic innovation clearly recognizable to researchers / as Ibero-American science education.
\end{abstract}

Investigación realizada en GEHyD-Grupo de Epistemología, Historia y Didáctica de las Ciencias Naturales, CeFIEC-Instituto de Investigaciones Centro de Formación e Investigación en Enseñanza de las Ciencias, Facultad de Ciencias Exactas y Naturales, Universidad de Buenos Aires. CeFIEC, 2 Piso, Pabellón 2, Ciudad Universitaria, Avenida Intendente Güiraldes 2160, (C1428EGA) Ciudad Autónoma de Buenos Aires, Argentina. 
Keywords: nature of science, philosophy of science, meta-sciences, scientific literacy, science curriculum, Ibero-America.

\section{Resumo}

O objetivo deste trabalho é apresentar os resultados de uma investigação que se refere a alguns acordos e desacordos sobre o conceito de "natureza da ciência" (conhecido internacionalmente com o NOS sigla) que aparecem entre os especialistas no ensino de ciências naturais; especificamente, vamos nos concentrar sobre aqueles da América Latina. Para fazer isso, consideramos a produção acadêmica na forma de artigos de periódicos, lançado entre 2000 e 2009, buscamos artigos que desenvolvem algum tipo de relação substantiva com os números da pesquisa. A principal conclusão deste estudo é que, hoje, o NOS é um campo de reflexão e inovação didática claramente reconhecível para pesquisadores / as Ibero-americano de educação científica.

Palavras-chave: natureza da ciência, epistemologia, metasciences, a alfabetização científica, currículo de ciências, a América Latina.

\section{Introducción}

Actualmente ¿sería suficiente con introducir en los planteamientos "renovadores" para la educación en ciencias las visiones -actualmente en boga- de la alfabetización científica y de las relaciones cienciatecnología-sociedad (CTS)? Dicho de otro modo, dirigir la educación hacia una formación científicotecnológica de la población general, ¿es adecuado para alcanzar los ambiciosos objetivos que la sociedad plantea para sus niños y niñas, adolescentes y jóvenes en los albores del siglo XXI? En este trabajo partimos de la tesis de que, a la hora de diseñar una genuina educación científica de calidad para todos $y$ todas, es necesario incluir en la enseñanza de las ciencias naturales algunos contenidos de la epistemología y de otras metaciencias (historia o sociología de la ciencia, principalmente), de los cuales hoy en día se nutren los investigadores del campo de la didáctica de las ciencias naturales. Estos contenidos forman parte de un "espacio" conceptual que los especialistas vienen denominando naturaleza de la ciencia (NOS, por sus siglas en inglés) (McComas, 1998; Flick y Lederman, 2002; Acevedo et al., 2005; Adúriz-Bravo, 2005a, 2005b).

Acudamos primero a lo que plantea el académico estadounidense William McComas (1998), quien conceptualiza la NOS como un campo de conocimiento "híbrido", que mezcla aspectos de varias metaciencias (esto es, ciencias que llevan a cabo una "metadiscusión" sobre las ciencias); él propone que las principales contribuciones provendrían de la epistemología, la historia y la sociología de la ciencia. Así, se podría entender la NOS como una línea de trabajo que emerge a partir de las necesidades curriculares que se establecen en los distintos niveles educativos (desde el inicial hasta el universitario), entendiendo que para la formación de ciudadanía, en la enseñanza de las ciencias naturales se han de combinar dos aspectos importantes -saber de ciencia y saber sobre ciencia.

En este trabajo nos apoyamos en una determinada conceptualización de la NOS: la entendemos como un conjunto de contenidos metacientíficos con valor para la educación científica (Adúriz-Bravo, 2009a, 2009b). Bajo esta perspectiva, en la NOS se privilegiaría la transposición de modelos que se generan en la epistemología erudita, con el objeto de que los ciudadanos y ciudadanas puedan generar respuestas informadas a las siguientes preguntas: ¿qué es la ciencia?, ¿cómo se transforma a lo largo de la historia? y ¿cuáles son sus relaciones con el contexto que la enmarca? (Adúriz-Bravo, 2005b).

Nuestro propósito es examinar las representaciones de NOS que se exponen en artículos especializados (papers) de la didáctica de las ciencias naturales, en el espacio geográfico de Iberoamérica. Lo anterior 
con el fin de determinar si hay o no consenso en esta temática, entre quienes hacen parte de nuestra comunidad iberoamericana de didactas. Bajo este panorama, nuestra intensión es revisar cuáles son las ideas que orientan la construcción de una naturaleza de la ciencia en la didáctica de las ciencias y cómo se asume la relación entre nuestra disciplina y las metaciencias; además de indagar por los posibles vínculos que se establecen entre la NOS y otras líneas de investigación didáctica.

\section{Aproximación a un marco conceptual}

Quienes investigamos e innovamos debemos adherir los productos derivados de la actividad científica a una postura epistemológica reconocible, que exprese nuestras convicciones acerca de dicha actividad y sus implicaciones sociales. Solo así, podremos generar un cambio en la enseñanza de las ciencias, en la formulación de los currículos, en las concepciones del profesorado y en otros campos de interés actual para la didáctica de las ciencias naturales.

Las posiciones epistemológicas -a menudo híbridas o tácitas- a las cuales acudimos los especialistas en didáctica de las ciencias naturales, podrían ubicarse en tres grandes "épocas" de la epistemología (Adúriz-Bravo, 2004): 1) el positivismo lógico y la concepción heredada; 2) el racionalismo crítico y la nueva filosofía de la ciencia; y 3 ) el posmodernismo y las visiones contemporáneas. Nos interesa recuperar aquí esta periodización "gruesa" de los momentos institucionalizados de la epistemología, debido a su capacidad de "mapear" las concepciones sobre la ciencia que tenemos los didactas.

Con respecto a la historia de la ciencia (concebida como metaciencia) y para los fines de este trabajo, concordamos con lo que plantean Camacho y Quintanilla (2008). Estos teóricos proponen ver la actividad científica como una construcción social que se desarrolla en el seno de unas relaciones culturales; ellos afirman que ni el descubrimiento ni la justificación del conocimiento científico se pueden mirar de manera "ahistórica" en la educación en ciencias. Estos autores señalan, desde lo que llaman una "posición naturalista pragmática", que la historia de la ciencia se podría entender como una "imagen cartográfica" de la ciencia: una especie de "mapa físico" en el que los objetos modelados solo existen en relación con los otros objetos que forman parte del sistema representado; por tanto, los datos no tienen significado de manera aislada en las comunidades científicas, sino relacionados entre ellos y con los modelos disponibles. Esta trama se da en función de las concepciones y finalidades de cada momento histórico. La historia de la ciencia, asumida desde esta posición "diacrónica", considera el contexto social, político, económico y cultural a partir del cual se postulan y desarrollan los conocimientos científicos.

\section{Metodología}

A continuación se presenta el proceso metodológico que realizamos para aproximarnos al estudio de la producción iberoamericana en torno a la NOS (ver figura 1). Tal proceso se organiza en dos fases.

La intención principal de nuestro estudio, fue la de caracterizar algunas representaciones de los investigadores en didáctica de las ciencias naturales con respecto al concepto de NOS. Para tal propósito, se decidió acotar el trabajo en tiempo y espacio, restringiéndolo a la producción aparecida entre 2000 y 2009 en Iberoamérica (España, Portugal y algunos países hispanos y luso-hablantes de América Latina y el Caribe). Para la colección del corpus de datos a analizar, elegimos textos bajo la forma de artículos de investigación, publicados en revistas especializadas de didáctica de las ciencias naturales de la región seleccionada. Las revistas consultadas fueron Revista Eureka sobre Enseñanza y Divulgación de las Ciencias (España), Enseñanza de las Ciencias (España), Revista Electrónica de Enseñanza de las Ciencias (España), Revista de Enseñanza de la Física (Argentina), Ciência \& Educação (Brasil), Investigações em Ensino de Ciências (Brasil) y Tecné, Episteme y Didaxis (Colombia), debido a que se encuentran entre las más prestigiosas y difundidas de la región.

Luego de esta selección, se recogieron todos aquellos artículos del corpus que en el título, resumen o palabras claves enunciaran los conceptos de naturaleza de la ciencia, epistemología, filosofía de la(s) 


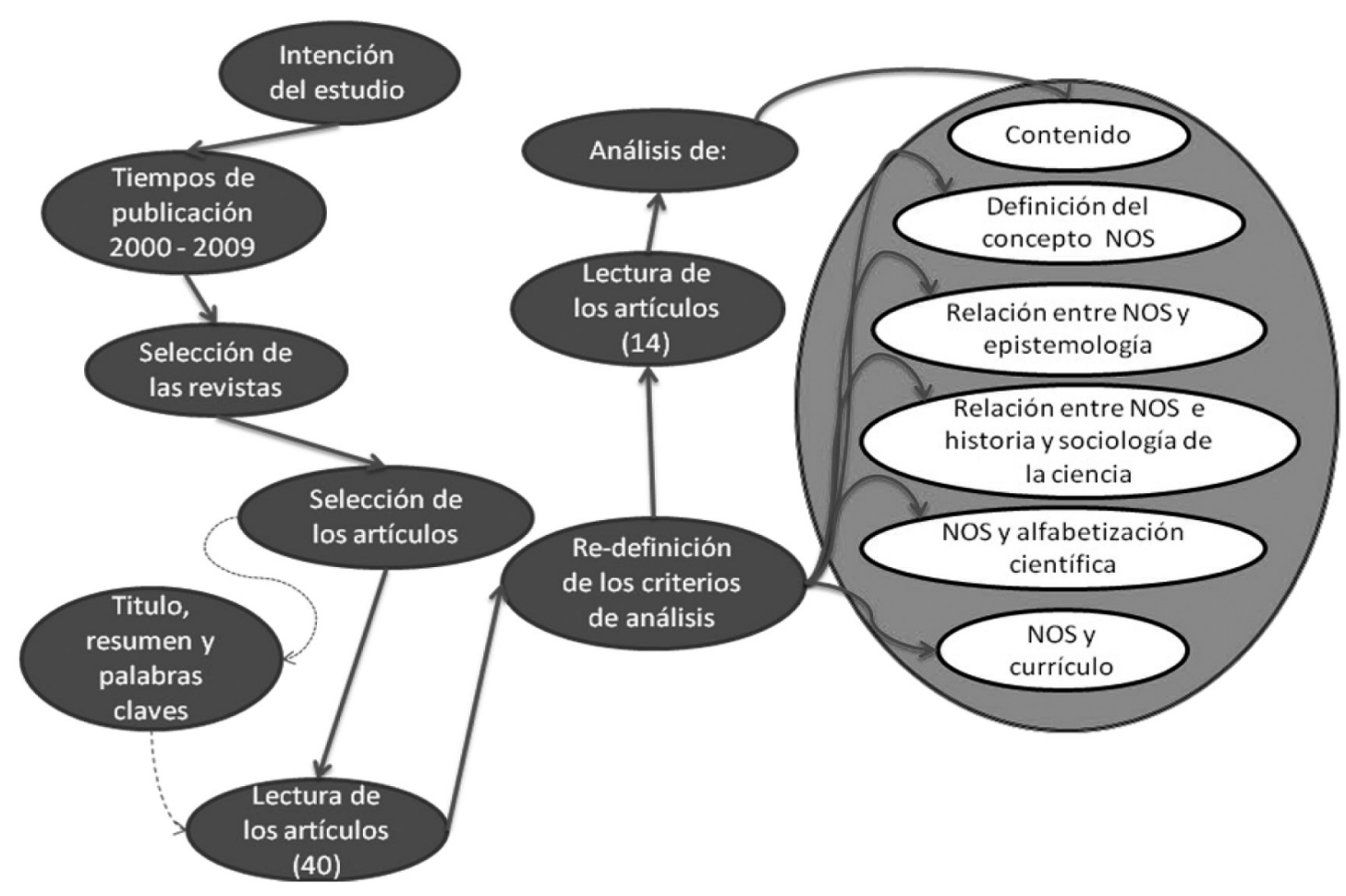

Figura 1. Elementos pragmáticos de nuestro estudio

Fuente: elaboración propia

ciencia(s), historia de la(s) ciencia(s) o incluso derivados de ellos. Con estos criterios obtuvimos cuarenta (40) artículos, que conformaron la primera fase del estudio. Al realizar la lectura de los cuarenta documentos, decidimos redefinir los criterios de análisis, afinándolos. En este sentido, no nos resultó suficiente con que se nombraran los conceptos antes mencionados; buscábamos también la enunciación de relaciones sustantivas. En consecuencia, una segunda lectura más específica nos permitió crear los siguientes criterios "de corte": definición del concepto de NOS; relaciones entre NOS y epistemología (filosofía de la ciencia); relaciones entre NOS e historia y sociología de la ciencia; NOS y alfabetización científica; y NOS y currículo. Con estos nuevos criterios realizamos una segunda selección de artículos, es decir, de los cuarenta originales quedaron catorce (14), y sobre estos realizamos un análisis de contenido (segunda fase del estudio).

En el cuadro 1 se referencian, únicamente, los textos de la primera fase del estudio, que quedaron eliminados en la segunda. Estos veintiséis (26) textos, hacen parte de los cuarenta (40) que habíamos seleccionado con los criterios "gruesos".

\section{Artículos de la primera fase únicamente (se reseñan en el Apéndice)}

Campanario y Otero, 2000; Medeiros y Bezerra Filho, 2000; Concari, 2001; De Mello Arruda, Rodrigues da Silva y Laburú, 2001; Furió, Vilches, Guisasola y Romo, 2001; Guridi y Salinas, 2001; Villani, 2001; Costa y Domènech, 2002; Marín Martínez, 2003; Paruelo, 2003; Colombo de Cudmani y Salinas de Sandoval, 2004; Rosado y García Carmona, 2005; Cornejo, 2006; Guridi, Salinas y Villani, 2006; Justi, 2006; Pecharromán y Pozo, 2006; Acevedo-Díaz, 2007; Banet, 2007; Gallego Torres y Gallego Badillo, 2007; Campello Queiroz y Almeida Barbosa-Lima, 2007; Vázquez Bernal, Jiménez Pérez y Mellado Jiménez, 2007; Camacho y Quintanilla, 2008; Henao y Stipcich, 2008; Marinho Oki y Fortuna de Moradillo, 2008; Vázquez y Manassero, 2008; Viau, Moro, Zamorano y Gibbs, 2008.

Cuadro 1. Artículos que pertenecen solo a la primera fase de nuestro estudio, y que fueron descartados para la segunda 
En el cuadro 2, se presentan las referencias que pertenecen a la segunda fase del estudio. En otras palabras, de todos los artículos que conformaron la primera fase, se seleccionaron catorce (14), a partir de los cuales se realizó el análisis de contenido.

\begin{tabular}{|l|}
\hline $\begin{array}{c}\text { Artículos que pasaron a la segunda fase } \\
\text { (se reseñan en el Apéndice) }\end{array}$ \\
\hline Arriassecq y Greca, 2002; Fernández, Gil, Carrascosa, \\
Cachapuz y Praia, 2002; Martín Díaz, 2002; Colombo de \\
Cudmani, 2003; Guridi y Arriassecq, 2004; Acevedo-Díaz, \\
Vázquez, Martín, Oliva, Acevedo, Paixão y Manassero, \\
2005; Adúriz-Bravo, 2005a; Viau, Zamorano, Gibbs y Moro, \\
2006; Acevedo-Díaz, Vázquez-Alonso, Manassero-Mas y \\
Acevedo-Romero, 2007a; Acevedo-Díaz, Vázquez-Alonso, \\
Manassero-Mas y Acevedo-Romero, 2007b; Guisasola y \\
Morentin, 2007; Praia, Gil-Pérez y Vilches, 2007; Acevedo- \\
Díaz, 2008; Fernández-González, 2008. \\
\hline
\end{tabular}

Cuadro 2. Artículos con los que nos quedamos para la segunda fase de nuestro estudio

\section{Análisis de resultados}

Se presentan enseguida los datos obtenidos y su respectivo análisis. En esta primera parte se muestra un panorama general del estudio, la procedencia de los autores (principales) y el número de artículos de la muestra que corresponde a cada revista consultada.

En las dos gráficas de la figura 2 se presentan las nacionalidades de los autores, para lo cual se eligió el autor principal de los artículos seleccionados como insumo para la investigación en la primera y segunda fase.
La figura 2 muestra los autores de cada nacionalidad. Se observa que, tanto en la primera como en la segunda fase, los investigadores con mayor interés en el campo de la NOS son españoles: quince (15) y ocho (8), respectivamente. La segunda comunidad de didactas de las ciencias naturales en el campo NOS más representada en las revistas que seleccionamos para el estudio es la de Argentina: aparecen diez (10) autores en la primera fase y cuatro (4) en la segunda. Por tanto, en esta línea de investigación, y con los parámetros acotados de la muestra, el mayor interés se da en España y Argentina, que producen, cada una, tantos o más artículos que el resto de los países en conjunto; esto convertiría a esos países en referentes para los desarrollos NOS regionales.

Se muestra a continuación la figura 3, que presenta el número de artículos que aportó cada revista a cada una de las fases del estudio realizado.

Los datos de la figura 3 indican que la comunidad que trabaja en NOS prefiere publicar en revistas españolas antes que en revistas pertenecientes a América Latina. Asimismo, los valores que arroja el análisis de la revista Eureka (para la primera fase, ocho (8) documentos; para la segunda, cinco (5) documentos) la posicionan como la publicación que más insumos aporta para nuestro estudio. Esto hace de Eureka, en los últimos años, un órgano privilegiado de difusión de resultados, innovaciones, reflexiones, etc., relacionados con la NOS.

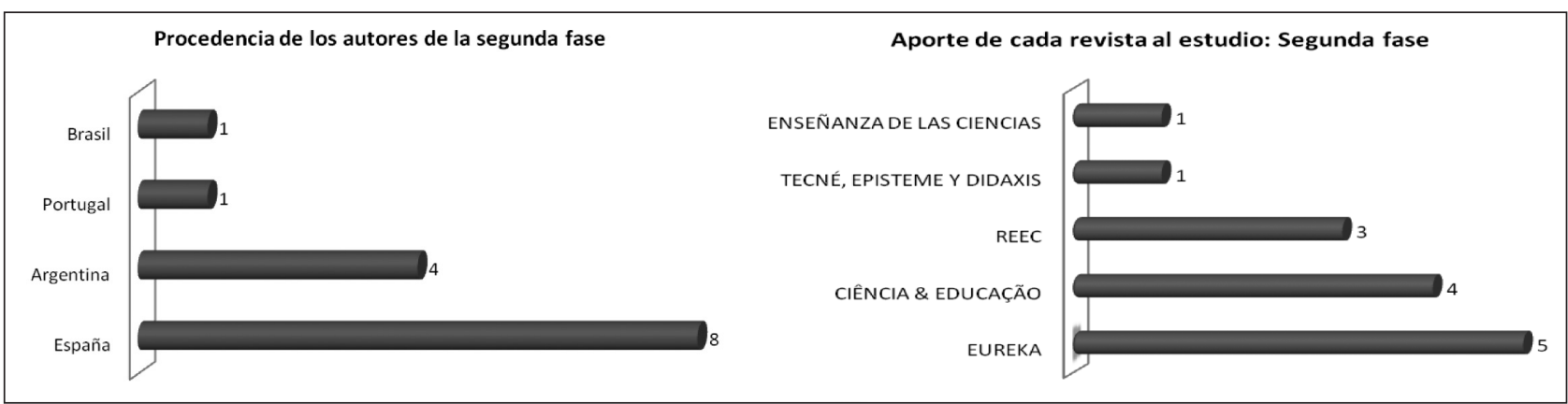

Figura 2. Procedencia de los autores principales de los artículos seleccionados como muestra para la primera y segunda fase del estudio

Fuente: elaboración propia 


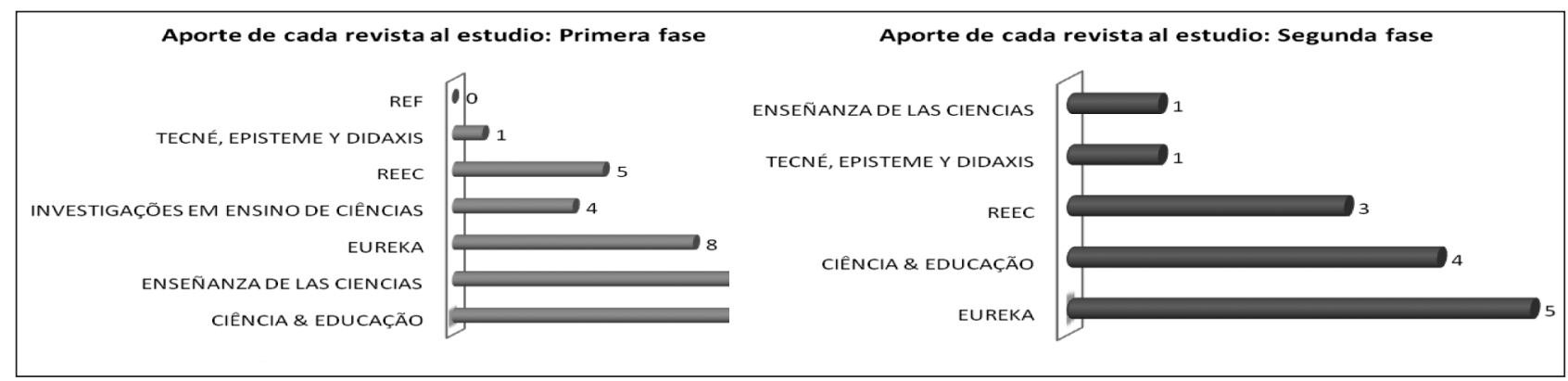

Figura 3. Aportes de cada revista a las muestras de la primera y segunda fase

Fuente: elaboración propia

La figura 4 reseña los conceptos a los cuales recurren los autores para generar el título de sus artículos.

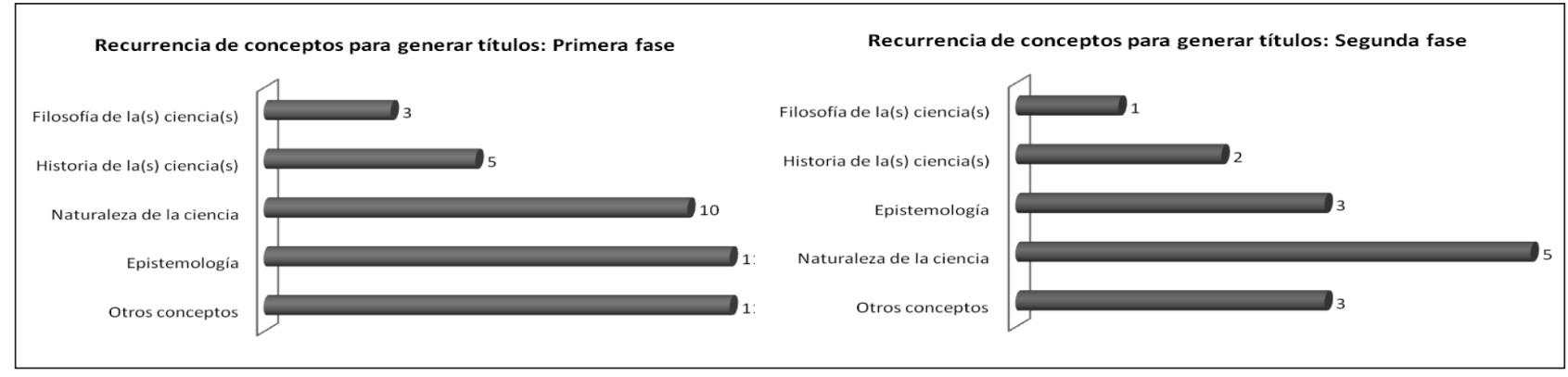

Figura 4. Conceptos que aparecen en los títulos de los artículos

Fuente: elaboración propia

En los artículos de la primera fase, los autores recurren a conceptos tales como epistemología, naturaleza de la ciencia, historia de la(s) ciencia(s) y filosofía de la(s) ciencia(s) para generar los títulos. Once (11) de los cuarenta documentos recurren a otros conceptos utilizados con baja frecuencia (por ejemplo: enseñanza, educación científica, ciencia, etc.). En lo que respecta a la segunda fase del estudio, el concepto al que más recurren los autores es el de naturaleza de la ciencia, seguido por el de epistemología. Esto guarda relación con lo anunciado en nuestro encuadre teórico: la comunidad de didactas se adhiere, en general, a la postura de que la metaciencia predominante en la NOS es la epistemología (o filosofía de la ciencia).

En el cuadro 3 se muestran, a grandes rasgos, los aspectos relevantes enunciados en el resumen de los documentos analizados para la segunda fase del estudio; la categorización resultó de la lectura de los 14 documentos que quedaron seleccionados.
Se estableció la frecuencia con que aparecían los enunciados en el apartado denominado resumen; el enunciado al que más acuden los autores es el de la "inclusión de la NOS en el currículo de ciencias", siendo al parecer una preocupación para los investigadores en didáctica de las ciencias. De la misma forma se determinaron los restantes enunciados, siendo el de menor recurrencia "consensos epistemológicos en didáctica”.

Aspectos relevantes enunciados en el resumen
1. Inclusión de la NOS en el currículo de ciencias.
2. Relación entre NOS y didáctica de las ciencias.
3. Relación entre NOS y alfabetización científica.
4. Incorporación de la epistemología en la enseñanza de
las ciencias.
5. Incorporación de la historia en la enseñanza de las
ciencias.
6. Consensos epistemológicos en didáctica.

Cuadro 3. Aspectos relevantes enunciados en los resúmenes de los trabajos 
En el cuadro 4 se presentan, de mayor a menor ocurrencia, las palabras clave que aparecen en los documentos analizados en la segunda fase del estudio. Como se puede observar, el concepto más recurrente es el de "naturaleza de la ciencia"; por tanto, se puede afirmar que este constructo tiene "entidad" en nuestro campo. En efecto, la gráfica de la derecha de la figura 4 , junto con los cuadros 3 y 4 , evidencia que los autores de la muestra de artículos presentan un interés por introducir la expresión "naturaleza de la ciencia” en el título, el resumen y las palabras clave, lo que indica el fortalecimiento de esta línea de trabajo en la comunidad iberoamericana de didáctica de las ciencias naturales.

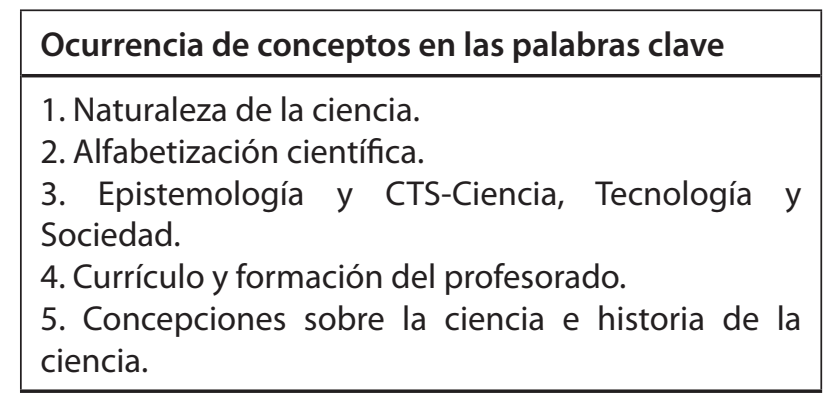

Cuadro 4. Ocurrencia de conceptos en las palabras clave de los trabajos

Además, y con forme a las discusiones que abordaremos más adelante, los resultados de este apartado nos permiten afirmar la idea del surgimiento de una sublínea de trabajo en NOS, que hemos denominado "vinculaciones de la NOS con el currículo de ciencias" (o, en forma abreviada: "NOS y currículo").

\section{Representaciones explícitas del concepto de "naturaleza de la ciencia" en la comunidad iberoamericana de didáctica de las ciencias naturales}

A continuación se presentan las definiciones "formales" de NOS encontradas en la muestra. Hemos tratado de ver en ellas las representaciones conceptuales de los investigadores en didáctica de las ciencias en Iberoamérica.
Acevedo-Díaz et al (2007a) sugieren que en la comunidad de didactas el concepto de NOS es dialéctico y complejo, lo que haría difícil definirlo con precisión y consenso. Una propuesta teórica, que viene sosteniendo este grupo de investigación, es asumir la NOS como un metaconocimiento sobre la ciencia que surge de las reflexiones interdisciplinares realizadas desde la filosofía, la historia y la sociología de la ciencia (Acevedo et al., 2005, 2007b; Acevedo-Díaz, 2008).

Una definición teóricamente más afinada es la que proponen Guisasola y Morentin (2007): la NOS comprende aspectos de diferentes áreas de conocimiento como la historia, la sociología y la filosofía de la ciencia con el propósito de explorar qué es la ciencia, cómo trabajan los científicos como grupo social y cómo la propia sociedad se enfrenta y reacciona ante los problemas derivados de la ciencia. Esta definición, junto con la de Acevedo-Díaz, al parecer, conforman las dos concepciones acerca de la naturaleza de la ciencia, construidas por los especialistas en didáctica de la ciencia, que tienen mayor acogida dentro de nuestra comunidad.

En otro texto, uno de nosotros caracteriza la NOS como un conjunto de contenidos metacientíficos con valor para la educación científica (AdúrizBravo, 2005a). Este tipo de definición amplia, podría ser conveniente por varias razones: en primer lugar, porque sitúa la naturaleza de la ciencia en el ámbito de acción de las metaciencias, que son disciplinas con carácter científico y, por tanto, las hace compatibles con las propias ciencias naturales y enseñables dentro de su espacio curricular. En segundo lugar, porque no separa estrictamente las diversas procedencias de las ideas a enseñar; estas provienen de la epistemología, la historia de la ciencia y la sociología de la ciencia principalmente; disciplinas entre las cuales una demarcación estricta es objeto de discusión, incluso entre sus propios especialistas. Y en tercer lugar, porque, al hablar de la voluntad profundamente educativa de la naturaleza de la ciencia, remite a genuinas transposiciones didácticas. 


\section{Aspectos de la NOS que deberían dirigir el trabajo de aula: sugerencias de los expertos}

En este apartado se presentan y caracterizan los aspectos de la NOS que, sugieren los especialistas en didáctica de las ciencias naturales, se deberían introducir en la enseñanza de las ciencias, para orientar el proceso de enseñanza del profesorado e incidir en el aprendizaje del estudiantado.
El cuadro anterior permite inferir dos cuestiones referentes a la inclusión de la NOS en la enseñanza de las ciencias. En primer lugar, los especialistas abogan por una reflexión en torno a los métodos que permiten validar el conocimiento científico, los valores involucrados en el desarrollo del conocimiento, las características que presenta la comunidad científica para el desarrollo del conocimiento, y la relación con la tecnología, la sociedad y la cultura. En segundo lugar, se propone que

\begin{tabular}{|c|c|}
\hline Aspectos & Afirmaciones \\
\hline Metodológico & $\begin{array}{l}\text { Métodos que validan el conocimiento científico (Adúriz-Bravo, 2005a; Acevedo et al., 2007b; } \\
\text { Acevedo, 2008). }\end{array}$ \\
\hline Axiológico & $\begin{array}{l}\text { Valores involucrados en las actividades de los hombres y mujeres de ciencias (Adúriz-Bravo, } \\
\text { 2005a; Acevedo et al., 2007b; Acevedo, 2008; Fernández-González, 2008). }\end{array}$ \\
\hline \multirow[b]{2}{*}{ De Interacción } & Relaciones con la tecnología (Acevedo et al., 2007b; Acevedo, 2008). \\
\hline & $\begin{array}{l}\text { Relaciones de la sociedad con el sistema tecnocientífico y los aportes que se generan a la cultura } \\
\text { y al progreso de la sociedad (Acevedo et al., 2007b; Acevedo, 2008). }\end{array}$ \\
\hline \multirow{6}{*}{ Teórico } & $\begin{array}{l}\text { Naturaleza y características de la comunidad científica (Arriassecq y Greca, 2002; Acevedo et al., } \\
\text { 2007b; Acevedo, 2008; Fernández-González, 2008). }\end{array}$ \\
\hline & $\begin{array}{l}\text { ¿Qué es la ciencia? (Arriassecq y Greca, 2002; Adúriz-Bravo, 2005a; Viau, Zamorano, Gibbs y Moro, } \\
\text { 2006; Acevedo et al., 2007b; Praia, Gil-Pérez y Vilches, 2007; Acevedo, 2008). }\end{array}$ \\
\hline & Funcionamiento interno y externo de la ciencia (Acevedo et al., 2007b; Acevedo, 2008). \\
\hline & $\begin{array}{l}\text { ¿Cómo se construye y se desarrolla el conocimiento?, (Arriassecq y Greca, 2002; Adúriz-Bravo, } \\
\text { 2005a; Viau, Zamorano, Gibbs y Moro, 2006; Acevedo et al., 2007b; Praia, Gil-Pérez y Vilches, 2007; } \\
\text { Acevedo, 2008; Fernández-González, 2008). }\end{array}$ \\
\hline & $\begin{array}{l}\text { La NOS incluye cuestiones filosóficas, sociológicas y psicológicas de la ciencia (Acevedo, 2008). } \\
\text { La NOS es principalmente una reflexión de tipo filosófico de la ciencia, ambientada en la historia } \\
\text { de la ciencia y advertida por la sociología de la ciencia contra el dogmatismo y el triunfalismo del } \\
\text { relato positivista tradicional (Adúriz-Bravo, 2005a). }\end{array}$ \\
\hline & $\begin{array}{l}\text { La NOS pretende construir una imagen de ciencia realista y racionalista moderada, de tal forma } \\
\text { que se puedan destacar los notables logros intelectuales y materiales de las ciencias naturales } \\
\text { sin rehuir a la discusión de sus limitaciones y de sus aspectos éticos o "humanos" (Adúriz-Bravo, } \\
\text { 2005a). }\end{array}$ \\
\hline \multirow{2}{*}{ Didáctico } & Sintonizar con los contenidos disciplinares, pedagógicos y didácticos (Adúriz-Bravo, 2005). \\
\hline & $\begin{array}{l}\text { ¿Cuál es la NOS que se quiere enseñar?, ¿para qué queremos enseñar NOS? (Acevedo et al, 2005, } \\
\text { 2007a; Adúriz-Bravo, 2005a; Praia et al., 2007). }\end{array}$ \\
\hline
\end{tabular}

Cuadro 5. Aspectos de la NOS que, según los especialistas, se deberían considerar para el trabajo de aula. 
los y las didactas de las ciencias generen una solución a los siguientes cuestionamientos, que han de surgir a la hora de una discusión-formulaciónimplementación de una estrategia didáctica: ¿qué tipo de ciencia se trabaja cuando se la "atraviesa" de NOS?, ¿qué NOS enseñar?, ¿para qué enseñar NOS? y ¿cuáles son los consensos sobre NOS que hoy en día ha validado la comunidad? Lo anterior nos lleva a pensar que en la actualidad no habría un consenso referente a este campo, entre todos los especialistas en didáctica.

Un aspecto de interés que surge a partir de lo encontrado en la lectura de los documentos es que la comunidad iberoamericana de didáctica de las ciencias naturales propone como componentes principales de la NOS la epistemología, la historia de la ciencia y la sociología de la ciencia; si bien esto parece un consenso extendido, algunos autores cambian la historia de la ciencia por la psicología de la ciencia (aunque la argumentación conceptual y metodológica para el cambio no queda completamente clara en los textos).

Por otra parte, Acevedo et al (2007a) aseguran que los investigadores en didáctica de las ciencias analizan y discuten a menudo sus representaciones de la NOS, las cuales son tan dinámicas y complejas como el propio conocimiento científico; ello origina una gama de concepciones acerca de la NOS, con distinciones desde el punto de vista conceptual y metodológico. Así, estamos en presencia de una multiplicidad de representaciones de la NOS en competencia al interior de la comunidad.

Por ejemplo, Acevedo et al (2005) consideran que la inclusión de la NOS en la enseñanza de las ciencias es un objetivo poco razonable pues, por su complejidad, la comprensión de la NOS podría quedar fuera del alcance de la mayoría del estudiantado, en particular en los primeros niveles educativos. Si la NOS se inserta en esos niveles, el objetivo no habría de ser centrarse tanto en la filosofía o en la sociología de la ciencia, como si se tratase de formar a los estudiantes para llegar a ser especialistas en estos campos del conocimiento, sino más bien ayudarles a comprender mejor cómo funcionan la ciencia y la tecnología contemporáneas.
Frente a esto, nuestra posición al respecto es que la naturaleza de la ciencia tiene tres grandes "finalidades" en la didáctica de las ciencias (AdúrizBravo, 2005a):

Finalidad intrínseca. La naturaleza de la ciencia ha de ser una reflexión "racional y razonable" sobre las propias ciencias, que sirva para analizarlas críticamente desde un segundo nivel de discurso.

Finalidad cultural. Se puede trabajar la naturaleza de la ciencia desde distintas áreas curriculares (por ejemplo, las ciencias naturales junto con la epistemología, la historia, las ciencias sociales, la matemática y la tecnología), para destacar su valor histórico como creación intelectual humana, situando personajes e ideas en el contexto social y amplio de cada época.

Finalidad instrumental. La naturaleza de la ciencia ha mostrado ser una herramienta valiosa para mejorar la enseñanza y el aprendizaje de los contenidos científicos. Las metaciencias son fuente de advertencias y consejos para identificar y atacar los obstáculos didácticos más importantes que aparecen asociados a la enseñanza de los grandes modelos de la historia de la ciencia. Un sólido conocimiento metacientífico permitiría que los y las estudiantes vinculen mejor los contenidos y formas de pensar de las ciencias con el conocimiento del sentido común. Y, por otra parte, la reflexión generada desde la epistemología (específicamente desde una postura realista y racionalista moderada) proveería de herramientas, materiales $\mathrm{y}$ enfoques útiles para nuestra tarea cotidiana de enseñar ciencias en el aula.

\section{Una propuesta de sublíneas de trabajo al interior de la NOS}

En esta sección presentamos y discutimos algunas de las ideas expresadas por los autores de los textos que se analizaron en la segunda fase del estudio; seleccionamos esas ideas porque -en nuestro opinión- tienen potencia teórica para la línea de trabajo NOS. Así mismo, nuestro estudio nos ha permitido reconocer algunas sublíneas al interior de la NOS, que creemos importantes para los 
desarrollos teóricos y metodológicos que se vienen adelantando en la actualidad.

\section{Relación entre NOS y epistemología}

Los investigadores afirman que las diversas reformas a la educación científica resaltan la importancia de desarrollar una imagen de la ciencia más válida desde el punto de vista epistemológico, que sea más coherente con las prácticas científicas actuales (Fernández et al., 2002; Acevedo et al., 2007b). A partir de lo anterior, la pregunta que surge es ¿qué concepción epistemológica asumir para esto? Acevedo-Díaz et al (2007b) afirman que en la actualidad se pueden encontrar epistemologías de todo tipo en las aulas de ciencias, por ejemplo: realismo, neopositivismo, funcionalismo, relativismo, y casi cualquier combinación posible de las anteriores, tal como racionalismo crítico, evolucionismo, realismo crítico, realismo constructivo, realismo perspectivo, realismo pragmático, empirismo constructivo, constructivismo sociológico, relativismo débil. Lo que preocupa a la comunidad, y aún necesita de argumentación in extenso, es cuál o cuáles de las anteriores posturas son valiosas para la didáctica de las ciencias, en coherencia con los marcos teóricos y las finalidades y valores sostenidos por nuestra disciplina.

Una discusión que presenta Acevedo (2008) es si la NOS debe restringirse a la inclusión de la epistemología en la educación científica, o si los contenidos deben ampliarse con aspectos esenciales de la sociología interna y externa de la ciencia, tal y como lo propugna el movimiento CTS para la enseñanza de las ciencias. Al respecto, Guisasola y Morentin (2007) consideran que la NOS se ocupa principalmente de los contenidos epistemológicos; estos se refieren a la forma en que la ciencia construye sus conocimientos, junto con los métodos, supuestos y creencias que la sustentan. Todo esto implica que existe una amplia variedad de aspectos que integran la NOS; de tal forma que no hay una única posición en lo referente a qué versión epistemológica seleccionar para infundirla en la NOS.

Acevedo y colaboradores (2007b) citan a filósofos como Eflin, Glennan y Reisch (1999) para presentar algunos de los presupuestos teóricos epistemológicos, asumidos en la NOS por los expertos. Por su parte, Guisasola y Morentin (2007) citan el Estudio de expertos de Osborne et al (2002) con el mismo fin. A continuación (cuadro 6), se presenta una matriz que compara las afirmaciones de estos dos trabajos en torno a lo conceptual, lo metodológico y la "correspondencia" con la realidad.

En la matriz se señalan algunas coincidencias frente a los presupuestos epistemológicos que se proponen trabajar en la enseñanza de las ciencias, al momento de integrar la NOS a una actividad de aula. Aparentemente, la inserción de la NOS en la enseñanza estaría soportada únicamente por la epistemología; este sin embargo es un aspecto no aceptado unívocamente en la comunidad de didactas de las ciencias.

\section{Relación entre NOS e historia y sociología de la ciencia}

Irene Arriassecq e Ileana María Greca (2002) afirman que la historia de la ciencia debería utilizarse en la enseñanza de las ciencias con varios propósitos: 1) para comprender las dificultades y obstáculos que se debieron superar para generar conocimiento científico y los contextos culturales, epistemológicos y tecnológicos, en los que se formuló una teoría o modelo científico diferente al actual; 2) para hacer ver que la ciencia es una actividad humana, realizada por hombres y mujeres que aportan contribuciones parciales frente a las preguntas que se plantean en cada época, o sea que rara vez hay "un solo descubridor"; y 3) para subrayar el hecho de que los científicos, en cada momento histórico, no pensaban en nuestros "términos actuales", ya que utilizaban las herramientas lógicas, metodológicas y epistemológicas de su contexto.

\section{NOS y alfabetización científica}

Algunos especialistas en didáctica de las ciencias naturales sostienen que para conseguir una participación ciudadana resulta necesaria la inclusión de la NOS en el currículo de las ciencias. No es esta la única razón posible para fundamentar la presencia curricular de la NOS, pero, según manifiestan 


\begin{tabular}{|c|c|c|c|}
\hline & & ACEVEDO ET AL. (2007b) & GUISASOLA Y MORENTIN (2007) \\
\hline \multirow{5}{*}{ 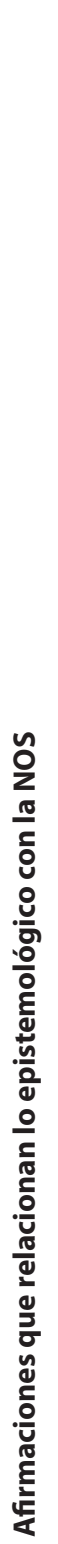 } & \multirow[b]{3}{*}{ 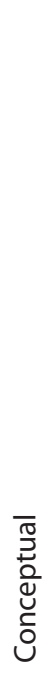 } & $\begin{array}{l}\text { El principal propósito de la ciencia es adquirir } \\
\text { conocimiento sobre el mundo físico. }\end{array}$ & $\begin{array}{l}\text { El papel de la ciencia es proporcionar explicaciones de los } \\
\text { fenómenos naturales; la ciencia está considerada como una dis- } \\
\text { ciplina para dirigir preguntas sobre el mundo natural. } \\
\text { La ciencia es una actividad que implica creatividad e imagi- } \\
\text { nación, así como otras muchas actividades humanas. Algunas } \\
\text { ideas científicas son grandes logros intelectuales. }\end{array}$ \\
\hline & & $\begin{array}{l}\text { La ciencia es cambiante, dinámica y } \\
\text { provisional. }\end{array}$ & $\begin{array}{l}\text { El trabajo de un científico supone un proceso continuo y } \\
\text { cíclico de hacer preguntas y buscar respuestas que conducen } \\
\text { a nuevas preguntas. Por tanto, el conocimiento científico es } \\
\text { tentativo (sujeto a cambios). }\end{array}$ \\
\hline & & $\begin{array}{l}\text { La generación del conocimiento científico } \\
\text { depende de compromisos teóricos y fac- } \\
\text { tores contextuales sociales e históricos } \\
\text { (contextualismo). }\end{array}$ & $\begin{array}{l}\text { La ciencia está inmersa en un contexto sociocultural y, por } \\
\text { tanto, está influenciada por los valores sociales y culturales, } \\
\text { por la subjetividad personal y por las conclusiones de los pro- } \\
\text { gramas de investigación. }\end{array}$ \\
\hline & $\begin{array}{l}\frac{0}{0} \\
\frac{0}{0} \\
\frac{0}{0} \\
\frac{0}{2} \\
\frac{0}{2}\end{array}$ & No existe un único método científico. & $\begin{array}{l}\text { La ciencia usa una metodología propia y la evidencia empírica } \\
\text { juega un papel importante, ya que diferencia la ciencia de otras } \\
\text { "formas de conocimiento". } \\
\text { La ciencia usa la evidencia empírica para comprobar las } \\
\text { ideas, pero el conocimiento científico no surge simplemente de } \\
\text { los datos sino de un proceso de interpretación y construcción } \\
\text { de teorías. Hay una distinción clara entre los datos experimen- } \\
\text { tales y las explicaciones. } \\
\text { La ciencia usa una gran variedad de métodos y no hay un } \\
\text { único método científico. } \\
\text { Los científicos desarrollan hipótesis y predicciones sobre } \\
\text { los fenómenos naturales, las cuales son comprobadas } \\
\text { empíricamente. }\end{array}$ \\
\hline & 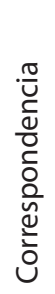 & $\begin{array}{l}\text { La verdad de las teorías científicas viene } \\
\text { determinada por aspectos del mundo que exis- } \\
\text { ten de modo independiente de los científicos } \\
\text { (realismo ontológico). } \\
\text { En el mundo hay un orden fundamental que la } \\
\text { ciencia pretende describir de la manera más } \\
\text { simple y comprensiva posible. }\end{array}$ & $\begin{array}{l}\text { El conocimiento científico actual es el mejor que tenemos } \\
\text { pero puede ser modificado en el futuro, debido a nuevas } \\
\text { interpretaciones de las evidencias o a nuevas evidencias. }\end{array}$ \\
\hline
\end{tabular}

Cuadro 6. Afirmaciones que vinculan epistemología con NOS en dos de los artículos analizados. Se subrayan coincidencias

los expertos, una mejor comprensión de la NOS permitiría tomar decisiones más razonadas sobre cuestiones públicas de carácter tecnocientífico, lo que probablemente contribuiría a hacer más factible la participación de los y las ciudadanas en estos asuntos (Acevedo et al., 2005). Por otro lado, Acevedo (2008) afirma que se necesita más investigación en didáctica para llegar a afirmar que una adecuada comprensión de la NOS realmente contribuye de un modo positivo a que el estudiantado y las personas adultas sean capaces de desarrollar actitudes más adecuadas hacia la ciencia. Al parecer, no hay un acuerdo entre los dos puntos de vista antes mencionados; esto hace pensar que aún no disponemos de una posición conceptual y metodológica única, a la hora de justificar por 
qué insertar la NOS en la educación. Este tópico entonces demandaría, de la comunidad en didáctica de las ciencias, la producción de resultados de investigación que aporten a la solución de esta controversia.

Desde otra perspectiva, Guridi y Arriassecq (2004) enuncian que el conocimiento de determinados tópicos de la epistemología, tales como evidencia, método y explicación, pueden ayudar a los ciudadanos a tomar decisiones más críticas acerca de importantes temas científicos y tecnológicos en las sociedades democráticas. Así, existe un consenso general con respecto a que es necesario que el ciudadano del siglo XXI no solo conozca la ciencia, sino que también sepa cómo es creada y validada, cómo se desenvuelve a través de la historia y cómo se relaciona con el medio social y cultural. Este sería el llamado enfoque CTS, que defiende la incorporación de la historia y la epistemología en la educación científica como parte de la formación de una conciencia ciudadana en el estudiantado de este nuevo milenio.

\section{NOS y currículo}

Acevedo et al (2005) señalan que los currículos de ciencias han estado centrados sobre todo en los contenidos conceptuales que se rigen por la lógica interna de la ciencia, y que por tanto se ha hecho a un lado la formación sobre la ciencia, esto es, sobre cuestiones tales como qué es la ciencia, cuál es su funcionamiento interno y externo; cómo se construye y desarrolla el conocimiento, qué métodos se usan para validarlo; cuáles son los valores implicados en las actividades científicas; cuál es la naturaleza de la comunidad científica; qué vínculos existen con la tecnología; cómo son las relaciones de la sociedad con el sistema tecnocientífico y viceversa; además de entender los aportes de la ciencia a la cultura y al progreso de la sociedad. Todos estos aspectos constituirían grosso modo lo que se conoce como NOS, entendida esta en un sentido amplio que trasciende lo exclusivamente epistemológico.

Por otra parte, Acevedo et al (2007a) afirman que, como elemento curricular novedoso dentro de la alfabetización científica y tecnológica, la enseñanza de la NOS es una innovación en la educación científica de extraordinaria magnitud, y no es fácil llevarla adelante. Postulan que habría dos corrientes opuestas sobre la dificultad de la NOS para convertirse en contenido curricular de la educación científica. La primera es una posición de disenso, que sostiene que no es factible alcanzar acuerdos básicos sobre NOS. La segunda es una posición de consenso, que defiende la posibilidad de conseguir acuerdos básicos sobre NOS, pese a su complejidad y a los debates, todavía existentes, en torno a ciertos temas. El desarrollo curricular de la NOS requiere, sin duda, una adaptación a la edad de los y las estudiantes, así como la correspondiente transposición didáctica y el diseño de actividades de enseñanza apropiadas para aclarar significados, conceptos, teorías, modelos, etc. Debido precisamente a la posibilidad de tener diversas transposiciones, la NOS como contenido educativo sería pertinente y válida para la mejora de la enseñanza y el aprendizaje de las ciencias.

Fernández-González (2008), por su parte, explora una relación más general entre NOS y currículo de ciencias. La NOS, como contenido curricular, ayudaría a:

1. Desarrollar una cultura científica para la participación ciudadana a través de cuestiones cotidianas y de repercusión social.

2. Conocer mejor el mundo y los grandes debates de la sociedad que conciernen a la ciencia, la tecnología y el medioambiente.

Acevedo Díaz (2008) recupera la pregunta estructurante de por qué es tan importante la NOS para el currículo de las ciencias. Y para construir una respuesta argumentada se apoya en la propuesta clásica de Rosalind Driver et al (1996), que define cinco valores para la NOS:

Utilitarista. Cuando los jóvenes comprenden la NOS, esto les permite tener cierta idea de qué es la ciencia y así manipular objetos y procesos tecnológicos de su vida cotidiana. 
Democrático. Cuando los jóvenes comprenden la NOS, se genera en ellos la capacidad de analizar $\mathrm{y}$ tomar decisiones bien informadas en cuestiones tecnocientíficas con interés social.

Cultural. Cuando los jóvenes comprenden la NOS, esta crea en ellos una valoración positiva de la ciencia; es decir, el estudiantado asume la ciencia como un elemento importante de la cultura contemporánea.

Axiológico. Cuando los jóvenes comprenden la NOS, esta les ayuda a entender mejor las normas y valores de la comunidad científica.

Docente. Cuando los jóvenes comprenden la NOS, se facilita el aprendizaje del contenido de las ciencias.

Algunos especialistas afirman que en la enseñanza actual de las ciencias se están tomando contenidos de naturaleza de la ciencia de un espectro más amplio de escuelas y autores, en consonancia con la voluntad de generar una reflexión con valor educativo, que se ajuste mejor a las poblaciones destinatarias. La tendencia, en nuestros días, es construir una naturaleza de la ciencia con libertad de criterios y pragmáticamente adecuada a las necesidades de la alfabetización científico-tecnológica, pero sin renunciar al rigor en la exposición de las ideas metacientíficas. Por eso, podríamos decir que ahora se busca dar respuesta a la pregunta referente a qué naturaleza de la ciencia hemos de enseñar en cada ámbito de formación específico (Adúriz-Bravo, 2005a).

\section{A modo de inferencias finales}

Como se ha pretendido mostrar en este documento, existiría un relativo acuerdo en torno a cuál es la "referencia" temática para el concepto de naturaleza de la ciencia, es decir, en torno a cuáles son las preguntas centrales que esta emergente componente curricular busca responder. Lo que no está tan claro aún son las contribuciones metaciencias específicas que conforman este nuevo campo de conocimiento, aspecto que exige de parte de la comunidad de didactas un trabajo hacia consensos pertinentes.
Estamos de acuerdo con la mayoría de los textos analizados en el hecho de que la naturaleza de la ciencia no sería únicamente una reflexión de carácter epistemológico, sino que también estaría acompañada por los análisis históricos y sociológicos de la ciencia. Sin embargo, podríamos afirmar que, en el corpus de artículos revisado para este trabajo, todavía no hay posiciones demasiado claras respecto a qué visiones de epistemología, historia de la ciencia y sociología de la ciencia elegir. Echamos en falta, entonces, más reflexión e investigación al respecto, a fin de determinar qué "épocas", ideas, autores o textos metacientíficos serían los más pertinentes para la enseñanza de las ciencias según el nivel educativo.

A la vista de los resultados, no resulta demasiado arriesgado afirmar que la comunidad española en didáctica de las ciencias está liderando los estudios en el campo de la naturaleza de la ciencia; en lo que se refiere a Sudamérica, los investigadores en didáctica de la Argentina son los que más interés prestan a esta línea de trabajo. Como mencionamos antes, el creciente interés no viene aún acompañado de acuerdos conceptuales y metodológicos extendidos.

El estudio que hemos realizado pretende poner en evidencia la existencia de nuevas sublíneas de análisis, ya reconocibles al interior de la NOS, como son las relaciones entre NOS, epistemología y otras metaciencias, o el vínculo de la NOS con el currículo de ciencias y la alfabetización científica. La existencia de estas sublíneas "atravesando" la producción iberoamericana reciente en NOS hablaría, a nuestro juicio, de la consolidación de la línea NOS al interior de la didáctica de las ciencias naturales.

\section{Bibliografía}

Acevedo Díaz, J. A. (2008). "El estado actual de la naturaleza de la ciencia en la didáctica de las ciencias". En Revista Eureka sobre Enseñanza y Divulgación de las Ciencias, vol. 5, núm. 2, pp. 134-169. [En línea].

Acevedo Díaz, J. A.; Vázquez Alonso, Á.; Martín, M.; Oliva, J. M.; Acevedo Romero, P.; Paixão, M. F. y Manassero Mas, M. A. (2005). 
"Naturaleza de la ciencia y educación científica para la participación ciudadana: Una revisión crítica”. En Revista Eureka sobre Enseñanza y Divulgación de las Ciencias, vol. 2, núm. 2, pp. 121-140. [En línea].

Acevedo Díaz, J. A.; Vázquez Alonso, Á.; Manassero Mas, M.A. y Acevedo Romero, P. (2007a). "Consensos sobre la naturaleza de la ciencia: Fundamentos de una investigación empírica". En Revista Eureka sobre Enseñanza y Divulgación de las Ciencias, vol. 4, núm. 1, pp. 42-66. [En línea].

Acevedo Díaz, J. A.; Vázquez Alonso, Á.; Manassero Mas, M.A, y Acevedo Romero, P. (2007b). "Consensos sobre la naturaleza de la ciencia: Aspectos epistemológicos”. En Revista Eureka sobre Enseñanza y Divulgación de las Ciencias, vol. 4, núm. 2, pp. 202-225. [En línea].

Adúriz-Bravo, A. (2004). "Apuntes sobre la formación epistemológica de los profesores de ciencias naturales". En Pedagogía y Saberes, núm. 21, pp. 9-19.

Adúriz-Bravo, A. (2005a). “QQué naturaleza de la ciencia hemos de saber los profesores de ciencias?: Una cuestión actual de la investigación didáctica”. En Tecné, Episteme y Didaxis, número extra, pp. 23-33.

Adúriz-Bravo, A. (2005b). Una introducción a la naturaleza de la ciencia: La epistemología en la enseñanza de las ciencias naturales. Buenos Aires: FCE.

Adúriz-Bravo, A. (2009). "La naturaleza de la ciencia "ambientada" en la historia de la ciencia”. En Enseñanza de las Ciencias, número extra "VIII Congreso Internacional sobre Investigación en Didáctica de las Ciencias", pp. 1178-1181. [En línea].

Amador Rodríguez, R. Y. y Adúriz-Bravo, A. (2009). "Una postura teórica para analizar la interacción entre la naturaleza de la ciencia y la didáctica de las ciencias". En Avances en educación en ciencia y tecnología: Enfoques $y$ estrategias, pp. 215-216. San Fernando del Valle de Catamarca: UNCa.
Arriassecq, I. y Greca, I.M. (2002). "Algunas consideraciones históricas, epistemológicas y didácticas para el abordaje de la teoría de la relatividad especial en el nivel medio y polimodal". En Ciência \& Educação, vol. 8, núm. 1, pp. 55-69. [En línea].

Camacho, J. y Quintanilla, M. (2008). “Resolución de problemas científicos desde la historia de la ciencia: retos y desafíos para promover competencias cognitivo lingüísticas en la química escolar". En Ciência \& Educação, vol. 14, núm. 2, pp. 197-212.

Colombo de Cudmani, L. (2003). “QQué puede aportar la epistemología a los diseños curriculares en física?" En Ciência \& Educação, vol. 9, núm. 1, pp. 83-91. [En línea].

Fernández-González, M. (2008). "La ciencia ayer y hoy: ciencias para el mundo contemporáneo. Algunas reflexiones didácticas". En Revista Eureka sobre Enseñanza y Divulgación de las Ciencias, vol. 5, núm. 2, 185-199. [En línea].

Flick, L. y Lederman, N. G. (2004). "Introduction". En L. Flick y N.G. Lederman (eds.), Scientific inquiry and nature of science: Implications for teaching, learning, and teacher education, IXXVII. Dordrecht: Kluwer.

Guridi, V. y Arriassecq, I. (2004). "Historia y filosofía de las ciencias en la educación polimodal: propuesta para su incorporación al aula". En Ciência \& Educação, vol. 10, núm. 3, 307-316. [En línea].

Guisasola, J. y Morentin, M. (2007). “¿Comprenden la naturaleza de la ciencia los futuros maestros y maestras de educación primaria?" En Revista Electrónica de Enseñanza de las Ciencias, vol. 6, núm. 2, pp. 246-262. [En línea].

Martín Díaz, M. J. (2002). “Enseñanza de las ciencias, ¿para qué?” En Revista Electrónica de Enseñanza de las Ciencias, vol. 1, núm. 2. [En línea].

McComas, W. (ed.). (1998). The nature of science in science education: Rationales and strategies. Dordrecht: Kluwer. 
Praia, J.; Gil Pérez, D. y Vilches, A. (2007). “O papel da natureza da ciência na educação para a cidadania”. En Ciência \& Educação, vol. 13, núm. 2, pp. 141-156. [En línea].

Fernández, I.; Gil, D.; Carrascosa, J.; Cachapuz, A. y Praia, J. (2002). "Visiones deformadas de la ciencia transmitidas por la enseñanza". En Enseñanza de las Ciencias, vol. 20, núm. 3, pp. 477-488.

Viau, J.; Zamorano, R. O.; Gibbs, H. y Moro, L. (2006). "Ciencia y pseudociencia en el aula: El caso del 'bosque energético"'. En Revista Electrónica de Enseñanza de las Ciencias, vol. 5, núm. 3, pp. 451-465. [En línea].

\section{Apéndice}

\section{Corpus de textos de la investigación}

Acevedo Díaz, J. A. (2007). "Las actitudes relacionadas con la ciencia y la tecnología en el estudio PISA 2006”. En Revista Eureka sobre Enseñanza y Divulgación de las Ciencias, vol. 4, núm. 3. [En línea].

Acevedo Díaz, J. A. (2008). "El estado actual de la naturaleza de la ciencia en la didáctica de las ciencias". En Revista Eureka sobre Enseñanza y Divulgación de las Ciencias, vol. 5, núm. 2. [En línea].

Acevedo Díaz, J. A.; Vázquez Alonso, Á.; Manassero Mas, M. A. y Acevedo-Romero, P. (2007). "Consensos sobre la naturaleza de la ciencia: Fundamentos de una investigación empírica". En Revista Eureka sobre Enseñanza y Divulgación de las Ciencias, vol. 4, núm. 1. [En línea].

Acevedo Díaz, J. A.; Vázquez Alonso, Á.; Manassero Mas, M. A. y Acevedo-Romero, P. (2007). "Consensos sobre la naturaleza de la ciencia: aspectos epistemológicos". En Revista Eureka sobre Enseñanza y Divulgación de las Ciencias, vol. 4, núm. 2. [En línea].

Acevedo Díaz, J. A.; Vázquez Alonso, Á.; Martín, M.; Oliva, J. M.; Acevedo, P.; Paixão M. F. y
Manassero, M. A. (2005). "Naturaleza de la ciencia y educación científica para la participación ciudadana: una revisión crítica”. En Revista Eureka sobre Enseñanza y Divulgación de las Ciencias, vol. 2, núm. 2. [En línea].

Adúriz-Bravo, A. (2005). “¿Qué naturaleza de la ciencia hemos de saber los profesores de ciencias?: Una cuestión actual de la investigación didáctica”. En Tecné, Episteme y Didaxis, número extraordinario "20 Congreso sobre Formación de Profesores de Ciencias", pp. 23-33.

Arriassecq, I y Greca, I. M. (2002). "Algunas consideraciones históricas, epistemológicas y didácticas para el abordaje de la teoría de la relatividad especial en el nivel medio y polimodal”. En Ciência \& Educação, vol. 8, núm. 1, pp. 55-69.

Banet, E. (2007). "Finalidades de la educación científica en secundaria: opinión del profesorado sobre la situación actual”. En Enseñanza de las Ciencias, vol. 25, núm. 1, pp. 5-20.

Camacho, J. y Quintanilla, M. (2008). “Resolución de problemas científicos desde la historia de la ciencia: retos y desafíos para promover competencias cognitivo lingüísticas en la química escolar". En Ciência \& Educação, vol. 14, núm. 2, pp. 197-212.

Campanario, J. y Otero, J. (2000). “Más allá de las ideas previas como dificultades de aprendizaje: las pautas de pensamiento, las concepciones epistemológicas y las estrategias metacognitivas de los alumnos de ciencias". En Enseñanza de las Ciencias, vol. 18, núm. 2.

Campello Queiroz, G. y Almeida Barbosa-Lima, M. (2007). "Conhecimento científico, seu ensino e aprendizagem: atualidade do construtivismo". En Ciência \& Educação, vol. 13, núm. 3, pp. 273-291.

Colombo de Cudmani, L. (2003). “QQué puede aportar la epistemología a los diseños curriculares en física?" En Ciência \& Educação, vol. 9, núm. 1, pp. 83-91. 
Colombo de Cudmani, L. y Salinas de Sandoval, J. (2004). “Es importante la epistemología de las ciencias en la formación de investigadores y de profesores en física?" En Enseñanza de las Ciencias, vol. 22, núm. 3.

Concari, S. (2001). "Las teorías y modelos en la explicación científica: implicancias para la enseñanza de las ciencias". En Ciência \& Educação, vol. 7, núm. 1, pp. 85-94.

Cornejo, J. (2006). “La enseñanza de la ciencia y la tecnología en la escuela argentina (1880-2000): un análisis desde los textos". En Enseñanza de las Ciencias, vol. 24, núm. 3.

Costa, A. y Domènech, G. (2002). “Distintas lecturas epistemológicas en tecnología y su incidencia en la educación". En Enseñanza de las Ciencias, vol. 20, núm. 1.

Fernández-González, M. (2008). “Ciencias para el mundo contemporáneo: algunas reflexiones didácticas". En Revista Eureka sobre Enseñanza y Divulgación de las Ciencias, vol. 5, núm. 2.

Fernández, I.; Gil, D.; Carrascosa, J.; Cachapuz, A. y Praia, J. (2002). "Visiones deformadas de la ciencia transmitidas por la enseñanza”. En Enseñanza de las Ciencias, vol. 20, núm. 3.

Furió, C.; Vilches, A.; Guisasola, J. y Romo, V. (2001). "Finalidades de la enseñanza de las ciencias en la secundaria obligatoria: ¿Alfabetización científica o preparación propedéutica?" En Enseñanza de las Ciencias, vol. 19, núm. 3, pp. 365-376.

Gallego Torres, A. y Gallego Badillo, R. (2007). "Historia, epistemología y didáctica de las ciencias: unas relaciones necesarias". En Ciência \& Educação, vol. 13, núm. 1, pp. 85-98.

Guisasola, J. y Morentin, M. (2007). “Comprenden la naturaleza de la ciencia los futuros maestros y maestras de educación primaria”. En Revista Electrónica de Enseñanza de las Ciencias, vol. 6 , núm. 2.
Guridi, V. y Arriassecq, I. (2004). "Historia y filosofía de las ciencias en la educación polimodal: propuesta para su incorporación al aula”. En Ciência \& Educação, vol. 10, núm. 3, pp. 307-316.

Guridi, V. y Salinas, J. (2001). "El vínculo entre aspectos conceptuales y epistemológicos en el aprendizaje de la física clásica”. En Investigações em Ensino de Ciências, vol. 6, núm. 2.

Guridi, V.; Salinas, J. y Villani, A. (2006). "Contribuciones de la epistemología de Laudan para la comprensión de concepciones epistemológicas sustentadas por estudiantes secundarios de física”. En Investigações em Ensino de Ciências, vol. 11, núm. 1.

Henao, B.L. y Stipcich, M.S. (2008). "Educación en ciencias y argumentación: la perspectiva de Toulmin como posible respuesta a las demandas y desafíos contemporáneos para la enseñanza de las ciencias experimentales". En Revista Electrónica de Enseñanza de las Ciencias, vol. 7, núm. 1.

Justi, R. (2007). "La enseñanza de ciencias basada en la elaboración de modelos". En Enseñanza de las Ciencias, vol. 24, núm. 2.

Marín Martínez, N. (2003). "Visión constructivista dinámica para la enseñanza de las ciencias". En Enseñanza de las Ciencias, número extra.

Marinho Oki, M. y Fortuna De Moradillo, E. (2008). “O ensino de história da química: contribuindo para a compreensão da natureza da ciencia”. En Ciência \& Educação, vol. 14, núm. 1, pp. 67-88.

Martín Díaz, M. J. (2002). "Enseñanza de las ciencias ipara qué?”. En Revista Electrónica de Enseñanza de las Ciencias, vol. 1, núm. 2.

Medeiros Severino, A. y Filho, B. (2000). "A natureza da ciência e a instrumentação para o ensino da física”. En Ciência \& Educação, vol. 6, núm. 2, pp. 107-117.

Mello Arruda, S.; Rodrigues Da Silva, M. y Laburú, C. E. (2001). "Laboratório didático de 
física a partir de uma perspectiva kuhniana". En Investigações em Ensino de Ciências, vol. 6, núm. 1.

Paruelo, J. (2003). "Enseñanza de las ciencias y filosofía”. En Enseñanza de las Ciencias, vol. 21, núm. 2.

Pecharromán, I y Pozo, J. I. (2006). “¿Cómo sé que es verdad?: Epistemologías intuitivas de los estudiantes sobre el conocimiento científico". En Investigações em Ensino de Ciências, vol. 11 , núm. 2.

Praia, J.; Gil-Pérez, D. y Vilches, A. (2007). "O papel da natureza da ciencia na educação para a cidadania”. En Ciência \& Educação, vol. 13, núm. 2, pp. 141-156.

Rosado, L. y García Carmona, A. (2005). "Razones didácticas y epistemológicas de la introducción de nociones de física de semiconductores en educación secundaria”. En Revista Electrónica de Enseñanza de las Ciencias, vol. 4, núm. 3.
Vázquez Bernal, B.; Jiménez Pérez, R. y Mellado Jiménez, V. (2007). "La reflexión en profesoras de ciencias experimentales de enseñanza secundaria: estudio de casos". Enseñanza de las Ciencias, vol. 25, núm. 1.

Vázquez, Á. y Manassero, A. (2008). "El declive de las actitudes hacia la ciencia de los estudiantes: un indicador inquietante para la educación científica”. En Revista Eureka sobre Enseñanza y Divulgación de las Ciencias, vol. 5, núm. 3.

Viau, J.; Moro. L.; Zamorano, R. y Gibbs, H. (2008). "La transferencia epistemológica de un modelo didáctico analógico". En Revista Eureka sobre Enseñanza y Divulgación de las Ciencias, vol. 5 , núm. 2.

Viau, J.; Zamorano, R. O.; Gibbs, H. y Moro, L. (2006). "Ciencia y pseudociencia en el aula: el caso del "bosque energético". En Revista Electrónica de Enseñanza de las Ciencias, vol. 5 , núm. 3 .

Villani, A. (2001). "Filosofia da ciência e ensino de ciência: uma analogía". En Ciência \& Educação, vol. 7, núm. 2, pp. 169-181. 